\title{
STAT5a-targeting miRNA enhances chemosensitivity to cisplatin and 5-fluorouracil in human colorectal cancer cells
}

\author{
XUAN HONG ${ }^{1}$, GONGYAN CHEN $^{1}$, MENG WANG ${ }^{1}$, CHANGJIE LOU $^{2}$, \\ YINLING MAO ${ }^{2}$, ZHIWEI $\mathrm{LI}^{2}$ and YANQIAO ZHANG ${ }^{2}$ \\ Departments of ${ }^{1}$ Respiratory Medical Oncology, and ${ }^{2}$ Gastrointestinal Medical Oncology, \\ The Third Affiliated Hospital of Harbin Medical University, Harbin, P.R. China \\ Received November 23, 2011; Accepted February 15, 2012
}

DOI: $10.3892 / \mathrm{mmr} .2012 .801$

\begin{abstract}
Signal transducers and activators of transcription 5 (STAT5) has been shown to be involved in a variety of cellular processes, including survival, proliferation, invasion, angiogenesis and immune evasion and is frequently overexpressed in human solid tumors and blood malignancies. The aim of this study was to investigate the role of STAT5a in colorectal cancer (CRC) progression. We inhibited the expression of STAT5a using lentivirus-mediated artificial microRNA (miRNA) interference in vitro and investigated the viability of CRC cells by CCK- 8 assay. We observed the cell viability after treatment with cisplatin (CDDP) or 5-fluorouracil (5-Fu) by CCK- 8 assay, and the apoptosis induced by chemotherapy using flow cytometric analysis and Annexin V RFP staining assay. We inhibited the mRNA expression by $54 \%$ and the protein expression by $60 \%$ of STAT 5 by RNA interference targeting STAT5a. Cell viability assays showed that inhibition of STAT5a did not affect the viability of SW1116 cells. However, we found that inhibition of STAT5a restored the sensitivity of SW1116 cells to CDDP and 5-Fu. In additional experiments, we found that inhibition of STAT5a significantly promoted $\mathrm{CRC}$ cell apoptosis by CDDP and 5-Fu. In the present study, we found that inhibition of STAT5a promotes apoptosis of CRC cells induced by chemotherapy drugs, such as CDDP or 5-Fu. These results suggest that inhibition of STAT5a may serve as a potential new target for CRC treatment.
\end{abstract}

\section{Introduction}

In the US, colorectal cancer (CRC) is the fourth most common cancer in both men and women, and new cases of CRC have been estimated at 102,900 in the US in 2010 (1). Although

Correspondence to: Dr Yanqiao Zhang, Department of Gastrointestinal Medical Oncology, The Third Affiliated Hospital of Harbin Medical University, No. 150 Haping Road, Harbin, P.R. China E-mail: dr_zhangyanqiao@yahoo.com.cn

Key words: signal transducers and activators of transcription 5a, colon cancer, apoptosis, cisplatin, 5-fluorouracil
CRC is the fifth most common malignancy in China, the incidence and mortality have been rapidly increasing in recent decades. Chemotherapy is one of the major strategies in CRC treatment, and cisplatin (CDDP) and 5-fluorouracil (5-Fu) are common chemotherapy drugs for CRC. It is known that CDDP and 5-Fu induce cell apoptosis and death via DNA damage in human colon cancer $(2,3)$, and combined therapy of CDDP and 5-Fu is the major regimen for CRC. Thus, it is necessary to develop new agents and methods including molecular targeting drugs. Therefore, understanding the function and role of tumor-associated genes is the basis for the development of new agents.

Signal transducers and activators of transcription (STATs) are an important family of transcription factors located in the cytoplasm and are activated by a variety of cytokines (4). This family of proteins comprises seven members - STAT1 to 4, STAT5a, STAT5b and STAT6 (5). STATs, especially STAT3 and STAT5, are an important point of convergence for many signaling pathways that are commonly activated in cancer cells. Both STAT3 and STAT5 have been shown to be involved in a variety of cellular processes, including survival, proliferation, invasion, angiogenesis and immune evasion and are frequently overactivated in human solid tumors and blood malignancies (6). Our previous study has shown that activated STAT5 is overexpressed in CRC and is related to poor prognosis (7). STAT5 is composed of two highly homologous isoforms, STAT5a and STAT5b, that are encoded by two separate genes with $96 \%$ similarity in their amino acid sequences (8). However, studies have revealed that STAT5a and STAT5b play different roles in the progression of various types of cancer cells. In glioblastoma multiforme (GBM) cells, research found that STAT5b, but not STAT5a, is involved in GBM cell growth, cell cycle progression, invasion and migration (9). Furthermore, activation of STAT5b increased hepatocellular carcinoma cell motility and invasion by induction of epithelial-mesenchymal transition (EMT) (10). In contrast, studies in human breast cancer revealed that overexpression of STAT5a reversed EMT progression and promoted differentiation of breast cancer cells (11).

In the present study, to understand the role of STAT5a in CRC progression, we inhibited expression of STAT5a using lentivirus-mediated artificial microRNA (miRNA) interference in vitro and investigated $\mathrm{CRC}$ cell viability. Although blocking 
of STAT5a did not directly inhibit the growth of cancer cells in CRC, subsequent results demonstrated that inhibition of STAT5a significantly promoted CRC cell apoptosis induced by treatment of CDDP and 5-Fu.

\section{Materials and methods}

Cell line and reagents. Human colorectal adenocarcinoma cell line, SW1116, and 293T cells were cultured in RPMI-1640 (HyClone, USA) medium and Dulbecco's modified Eagle's medium (DMEM) (HyClone), respectively, supplemented with $10 \%$ fetal bovine serum (HyClone), penicillin $(100 \mathrm{U} / \mathrm{ml})$ and streptomycin $(100 \mu \mathrm{g} / \mathrm{ml})$ (Invitrogen, USA) under standard culture conditions $\left(95 \%\right.$ air- $\left.5 \% \mathrm{CO}_{2}, 37^{\circ} \mathrm{C}\right)$. $\mathrm{CDDP}$ and 5 -Fu were purchased from F.H. Faulding and Co. (Australia).

Lentivirus for delivery of miRNA and transfection. Four different miRNA oligonucleotides targeting STAT5a were designed and synthesized by Shanghai R\&S Biotechnology Co. pLenti6.3-MCS/V5 DEST lentiviral vector was purchased from Shanghai R\&S Biotechnology Co. We constructed four lentivirus vectors targeting STAT5a that were named pLenti6.3-EGFP-Stat5-miR. 293T cells (Invitrogen) were co-transfected with lentivirus expression plasmid and packaging plasmids (Invitrogen). Transfections were carried out using Lipofectamine 2000 (Invitrogen). Interference efficacy was confirmed by qRT-PCR. According to interference efficacy, the miRNA targeting sequence of ACTTGCCGGGCTGGAACTACA was used in this study. SW1116 cells were infected with the recombinant lentivirus vectors at a multiplicity of infection (MOI) of 30 .

Quantitative real-time PCR. Total-RNA was isolated from SW1116 cells using the TRIzol reagent (Invitrogen). cDNA was synthesized from isolated RNA using the RevertAid ${ }^{\mathrm{TM}}$ First Strand cDNA Synthesis kit (MBI, USA) according to the manufacturer's instructions. The SYBR-Green qPCR SuperMix from Invitrogen was used. qRT-PCR was performed using the Eppendorf Realplex4 (Eppendorf). The CT value of mRNA expression for each sample was normalized with internal control gene $\beta$-actin and relative quantification values were plotted. The primersequences of each gene for real-timeqPCR were as follows: STAT5a, forward, 5'-GCTCCCTATAACATGTACCCACA-3' and reverse, 5'-CTGGCCACATCCATGGTC-3'; $\beta$-actin, forward, 5'-TCCTTCCTGGGCATGGAGT-3' and reverse, 5'-CAGGAGGAGCAATGATCTTGAT-3'. The reaction was performed at $50^{\circ} \mathrm{C}$ for $30 \mathrm{~min}, 95^{\circ} \mathrm{C}$ for $2 \mathrm{~min}, 95^{\circ} \mathrm{C}$ for 40 cycles of $20 \mathrm{sec}$ each, $58^{\circ} \mathrm{C}$ for $15 \mathrm{sec}$, and $72^{\circ} \mathrm{C}$ for $20 \mathrm{sec}$ with extension at $72^{\circ} \mathrm{C}$ for $10 \mathrm{~min}$.

Western blotting. The cells were lysed and protein extraction was performed. Protein concentration was determined using the Lowry method (Bio-Rad, USA). Extracted proteins were resolved on $8 \%$ SDS-PAGE, electrophoretically transferred onto nitrocellulose membranes (Bio-Rad). Membranes were incubated in blocking buffer (5\% nonfat dry milk, $0.1 \%$ Tween-20 and Tris-buffered saline) for $1 \mathrm{~h}$, then washed and probed with the primary antibodies. Membranes were washed with TBS-T, probed in the dark with the appropriated secondary antibody and then visualized using BCIP/NBT solution. Mouse monoclonal antibodies to STAT5 and mouse monoclonal antibodies to $\beta$-actin were purchased from Santa Cruz Biotechnology, Inc. (USA).

Cell proliferation assay. Cell viability and proliferation assays were performed using the Cell Counting Kit-8 (CCK-8; Beyotime, China) according to the manufacturer's protocol. Briefly, control cells, Lenti-control cells and Lenti-STAT5a cells were seeded in 96-well plates at an initial density of $5 \times 10^{3}$ cells/well. At $0,24,48$ and $72 \mathrm{~h}$ respectively, $10 \mu 1$ of CCK-8 solution was added and the plates were incubated for $3 \mathrm{~h}$ at $37^{\circ} \mathrm{C}$. Then absorbance at $450 \mathrm{~nm}$ was determined by a microplate reader (Thermo, USA). The absorbance of the culture medium plus CCK- 8 in the absence of cells was used as background control. Experiments were performed in triplicate.

Apoptosis detection assay. Cell apoptosis was determined by flow cytometric analysis, and the Annexin V RFP staining assay was performed in accordance with the manufacturer's protocol (Mbchem). Briefly, 5x10 cells were harvested. Floating and trypsinized adherent cells were collected at 24 and $48 \mathrm{~h}$ after treatment with CDDP or 5-Fu. They were resuspended in $195 \mu \mathrm{l}$ of binding buffer and $5 \mu \mathrm{l}$ Annexin V PE, and then incubated for $10 \mathrm{~min}$ in the dark at room temperature. An additional $200 \mu \mathrm{l}$ of binding buffer was added, and the analysis was immediately performed using a flow cytometer (BD Biosciences, USA).

Statistical analysis. The results are expressed as mean \pm standard deviation (SD). One-way analysis of variance (ANOVA) was used to determine the levels of difference between all groups. P-values $<0.05$ (two-sided) were considered to indicate statistical significance. SPSS statistical software (version 13.0) was used for all calculations and statistical analyses.

\section{Results}

mRNA and protein expression of STAT5a is inhibited after miRNA interference. Firstly, we tested the efficacy of lentivirus-mediated miRNA interference targeting STAT5a by qRT-PCR and western blotting. Results of qRT-PCR showed that mRNA expression of STAT5a was decreased by $54 \%$ at $72 \mathrm{~h}$ after miRNA interference (Fig. 1A). Similar to the downregulation of mRNA levels, the expression of STAT5a in the Lenti-STAT5a group was significant lower than that in the other control groups. A reduction in STAT5 protein level by approximately $60 \%$ was noted at $72 \mathrm{~h}$ (Fig. 1B).

Lentivirus-mediated miRNA interference targeting STAT5a does not affect SW1116 cell growth. To observe SW1116 cell viability, we performed CCK-8 assay at $0,24,48$ and $72 \mathrm{~h}$ after interference, respectively (Fig. 2A). However, miRNA interference targeting STAT5a failed to affect SW1116 cell growth at any time point when compared to the other control groups $(\mathrm{P}>0.05)$.

Inhibition of STAT5 a restores cancer cell sensitivity of SW1116 cells to CDDP and 5-Fu. To investigate whether lentivirusmediated miRNA interference targeting STAT5a affects cell viability after treatment with CDDP or 5-Fu, CCK-8 assay 
A

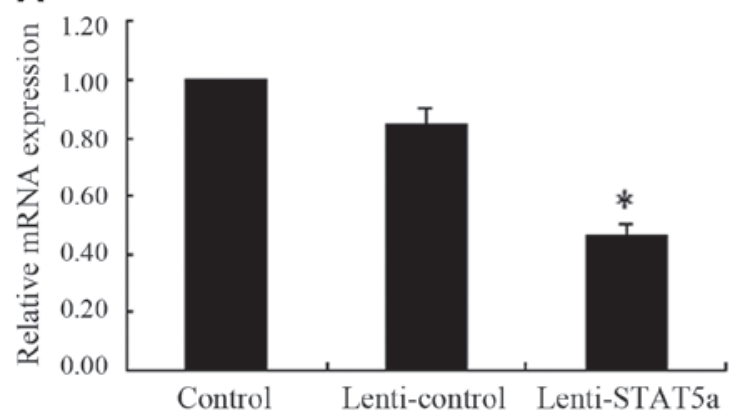

B

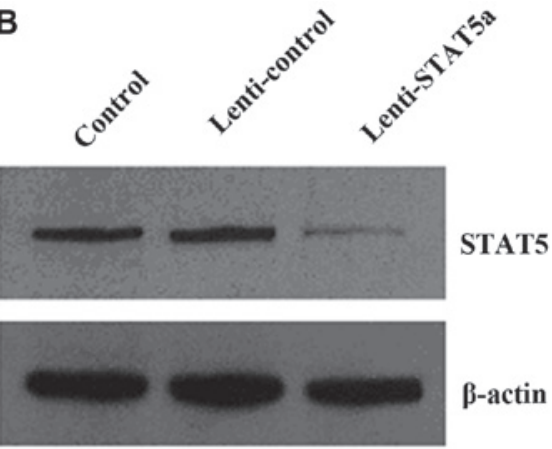

Figure 1. Lentivirus-mediated miRNA interference targeting STAT5a inhibits mRNA and protein levels of STAT5a in SW1116 cells. (A) mRNA expression of STAT5a was decreased by $54 \%$ at $72 \mathrm{~h}$ after miRNA interference in the Lenti-STAT5a group ("P<0.05). (B) Protein expression of STAT5a at $72 \mathrm{~h}$ after miRNA interference was decreased by approximately $60 \%$ in the Lenti-STAT5a group.
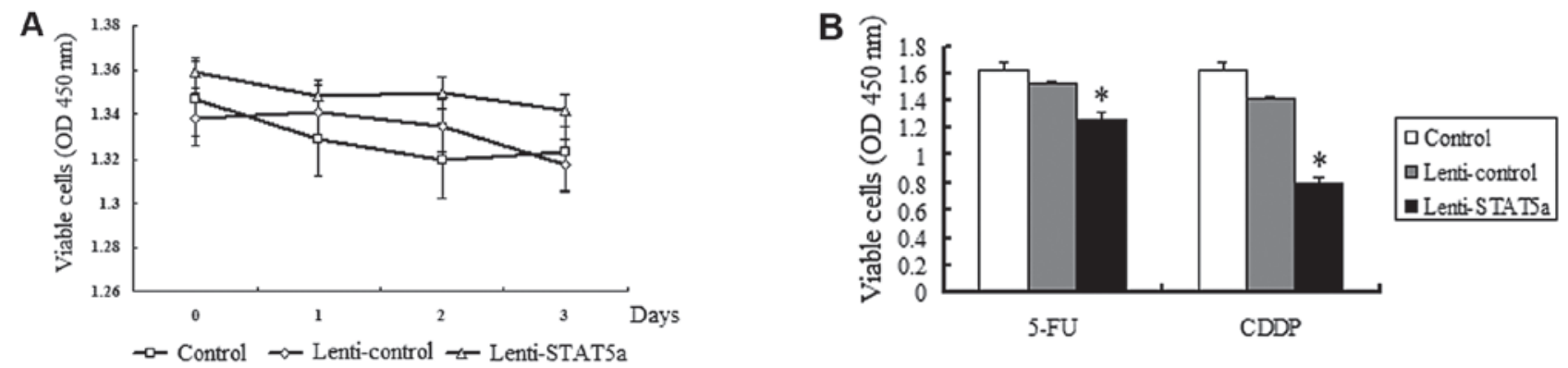

Figure 2. CCK-8 assay results showed inhibition of STAT5a failed to affect SW1116 cell viability, but affected cell viability after treatment with CDDP or 5-Fu . (A) CCK-8 assay was performed on days $0,1,2,3$ after interference. The viability curve indicates that no significant difference existed on days $0,1,2,3$ in the three groups $(\mathrm{P}>0.05)$. (B) Cells were treated with CDDP $(20 \mu \mathrm{g} / \mathrm{ml})$ or $5-\mathrm{Fu}(200 \mu \mathrm{g} / \mathrm{ml})$ and CCK-8 assay was performed after $24 \mathrm{~h}$. The interference of targeting STAT5a promoted inhibition of SW1116 cell growth after $24 \mathrm{~h}$ of treatment with CDDP or 5-Fu ("P<0.05).

was performed after cells were treated for $24 \mathrm{~h}$. The exposure concentration of CDDP and 5-Fu was 20 and $200 \mu \mathrm{g} /$ $\mathrm{ml}$, respectively. Results revealed that inhibition of STAT5 induced by interference targeting STAT5a promoted a CDDPor 5-Fu-induced decrease in cell viability compared with the two control groups $(\mathrm{P}<0.05)$. This finding demonstrated that inhibition of STAT5a sensitizes CRC cells to chemotherapy (Fig. 2B).

Inhibition of STAT5 a promotes apoptosis induced by CDDP or $5-F u$. To investigate whether the increased cell sensitivity of the CRC cells to CDDP or 5-Fu after STAT5a inhibition was due to apoptosis, apoptosis analysis was performed using flow cytometry 24 and $48 \mathrm{~h}$ after treatment with CDDP or 5-Fu (Fig. 3A). The cell apoptosis rates of the Lenti-STAT5a group (35.27 \pm 4.19 and $88.53 \pm 4.30 \%)$ at 24 and $48 \mathrm{~h}$ after treatment with CDDP were significantly higher than the rates of the control $(16.17 \pm 1.99$ and $58.95 \pm 2.11 \%)$ and Lenti-control groups $(18.49 \pm 2.97$ and $62.44 \pm 2.77 \%)(\mathrm{P}<0.05)$ (Fig. 3B). Similarly, an increase in apoptosis rates was also observed in the Lenti-STAT5a cells $(49.98 \pm 3.27$ and $70.26 \pm 2.89 \%)$ at 24 and $48 \mathrm{~h}$ after treatment with 5-Fu compared with the control $(20.58 \pm 3.19$ and $42.95 \pm 2.71 \%)$ and Lenti-control groups $(25.70 \pm 4.81$ and $46.70 \pm 4.97 \%)(\mathrm{P}<0.05)$ (Fig. 3C). The results indicate that inhibition of STAT5a promotes CRC cell apoptosis induced by DNA damage.

\section{Discussion}

As downstream signal transducers of the JAK-STAT pathway, persistent activation of STAT3 and STAT5 to the nucleus, binds specific DNA elements, activating a set of genes involved in malignant progression $(5,12-14)$. As STAT3 has been the focus of cancer research, STAT3 inhibitors are being investigated, and a growing number of preclinical studies are ongoing. Therefore, more and more researchers have begun to focus on STAT5 (15-19). Evidence reveals that inhibition of STAT5 affects biological behavior of cancer cells. Studies in vitro and in vivo have shown that STAT5a and STAT5b differentially regulate the motility and migration of human mammary carcinoma cells $(20,21)$, and inhibition of Stat $5 \mathrm{a} / \mathrm{b}$ by antisense oligonucleotides, RNA interference, or adenoviral expression of dominant-negative Stat $5 \mathrm{a} / \mathrm{b}$ can induce cell death of human prostate cancer cells (22).

In the present study, mRNA expression of STAT5 was inhibited by $54 \%$ and protein expression by $60 \%$ by miRNA targeting STAT5a interference. No inhibition of the viability of the CRC cells was observed. This is inconsistent with previous studies which demonstrated that inhibition by transient transfection of STAT5 siRNA suppressed growth, induced G1 cell cycle arrest and inhibited invasion of CRC cells (23). More recent research indicates that STAT5b expression is significantly higher than that of STAT5a in 
A
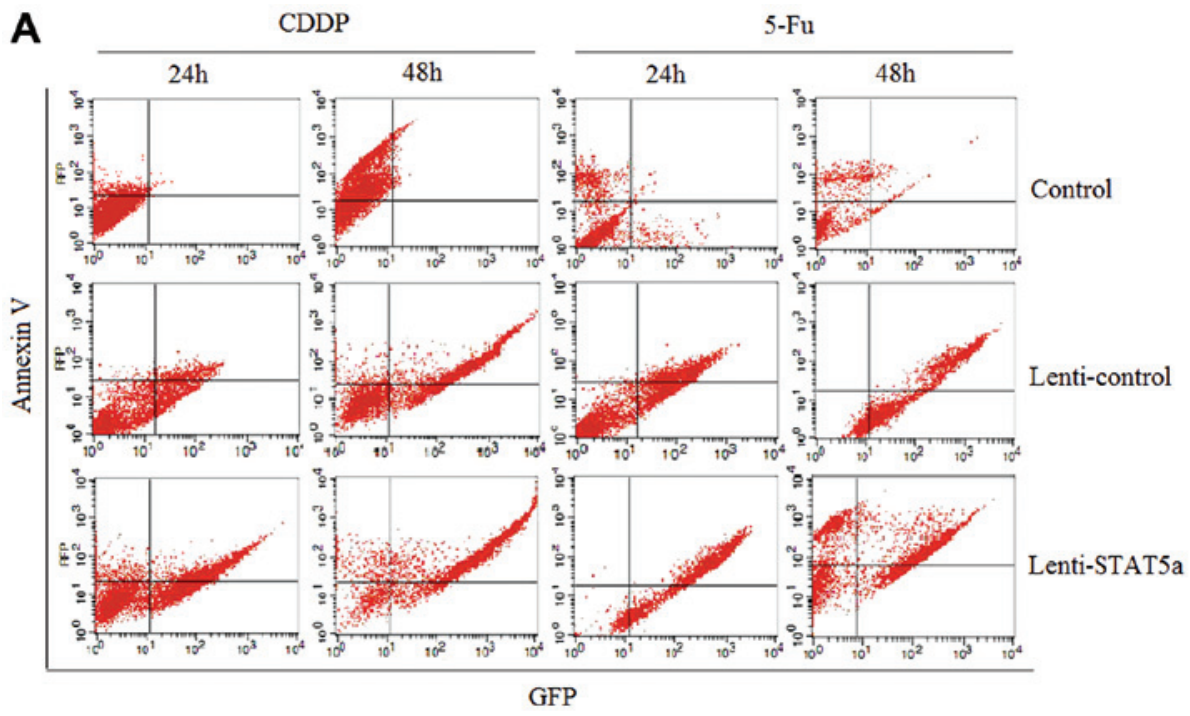

$5-\mathrm{Fu}$
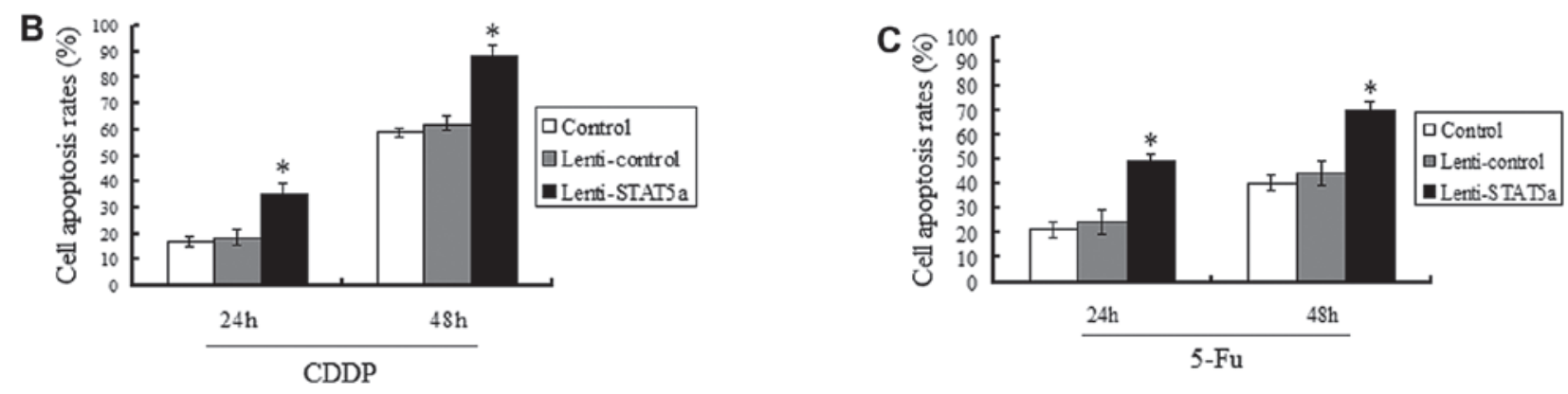

Figure 3. Inhibition of STAT5a enhances apoptosis induced by CDDP or 5-Fu. (A) Apoptosis rates were determined by Annexin V RFP staining assay and flow cytometric analysis at 24 and $48 \mathrm{~h}$ after treatment with CDDP or 5-Fu. (B) Cell apoptosis rates in the Lenti-STAT5a group both at 24 and $48 \mathrm{~h}$ after treatment with CDDP were significantly higher than the rates noted in the other control groups $($ P $<0.05)$. (C) Similarly, the cell apoptosis rates of the Lenti-STAT5a group at 24 and $48 \mathrm{~h}$ after treatment with $5-\mathrm{Fu}$ were significantly higher than the rates in the other control groups $\left({ }^{*} \mathrm{P}<0.05\right)$.

colorectal adenocarcinoma tissue and STAT5b affects CRC cell apoptosis to a greater extent than STAT5a even though both STAT5a and STAT5b are involved in cell growth, cell cycle progression and apoptosis (24). This may explain why STAT5b plays a more important role than STAT5a in the biological behavior of CRC cells. Moreover, we inhibited STAT5 expression by lentiviral vector-mediated miRNA interference which differed from previous studies. This may have caused differences in the findings.

Although inhibition of STAT5a did not affect the viability of SW1116 cells, inhibition of STAT5a sensitized the SW1116 cells to CDDP or 5-Fu treatment. Other studies have shown that STAT5 regulated the sensitivity of cancer cells to chemotherapy. Researchers found that high STAT5 levels mediate imatinib resistance in chronic myeloid leukemia (CML) (25); inhibition of STAT5 by siRNA significantly reduced the activities of multi-drug resistant protein and enhanced the sensitivity of CML cells to imatinib (26). Research on squamous cell carcinoma of the head and neck demonstrated that STAT5 activation was associated with resistance to cisplatinmediated apoptosis and growth inhibition induced by the epidermal growth factor receptor tyrosine kinase inhibitor, such as erlotinib (27). Moreover, researchers confirmed that STAT5 knockdown with STAT5-ASO significantly delays castration-resistant prostate cancer progression in vivo (28). In a subsequent experiment, we found that inhibition of STAT5a significantly promoted CRC cell apoptosis by chemotherapy. It is known that a defective apoptosis pathway is a major mechanism of drug resistance in cancer cells $(29,30)$. Therefore, we speculate that promotion of apoptosis may be the main reason why blockage of STAT5a enhanced the sensitivity of cancer cells to chemotherapy. STAT5a probably regulates other drug-resistance-related protein and takes part in resistance of colorectal cells to chemotherapy. Further studies are needed to understand the mechanism of STAT5a in the drug sensitivity of cancer cells.

In summary, inhibition of STAT5a promoted apoptosis of CRC cells induced by chemotherapy drugs, CDDP and 5-Fu. This study reveals a novel mechanism by which STAT5a restores the sensitivity of CRC cells to cytotoxic drugs. These results suggest that inhibition of STAT5a may serve as a potential new target for CRC treatment, and that it is possible that a STAT5a inhibitor combined with chemotherapy may be used in the future. It has been confirmed that the two main apoptotic pathways are the cytoplasmic and mitochondrial pathways (31). We plan to investigate the possible molecular pathway involved in promoting apoptosis induced by the inhibition of STAT5a. 


\section{References}

1. National Cancer Institute (NCI): NCI website, Colon and Rectal Cancer homepage. Available at: http://www.cancer.gov/cancertopics/types/colon-and-rectal. Accessed May 16, 2011.

2. Boulikas T and Vougiouka M: Cisplatin and platinum drugs at the molecular level. Oncol Rep 10: 1663-1682, 2003.

3. Lönn U, Lönn S, Nylen U and Winblad G: 5-Fluoropyrimidineinduced DNA damage in human colon adenocarcinoma and its augmentation by the nucleoside transport inhibitor dipyridamole. Cancer Res 49: 1085-1089, 1989.

4. Behrmann I, Smyczek T, Heinrich PC, et al: Janus kinase (Jak) subcellular localization revisited: the exclusive membrane localization of endogenous Janus kinase 1 by cytokine receptor interaction uncovers the Jak. receptor complex to be equivalent to a receptor tyrosine kinase. J Biol Chem 279: 35486-35493, 2004.

5. Darnell JE Jr: STATs and gene regulation. Science 277: 1630-1635, 1997.

6. Yu H and Jove R: The STATs of cancer - new molecular targets come of age. Nat Rev Cancer 4: 97-105, 2004.

7. Mao YL, Li ZW, Lou CJ, et al: Phospho-STAT5 expression is associated with poor prognosis of human colonic adenocarcinoma. Pathol Oncol Res 17: 333-339, 2011.

8. Liu X, Robinson GW, Gouilleux F, et al: Cloning and expression of Stat5 and an additional homologue (Stat5b) involved in prolactin signal transduction in mouse mammary tissue. Proc Natl Acad Sci USA 92: 8831-8835, 1995.

9. Liang QC, Xiong H, Zhao ZW, et al: Inhibition of transcription factor STAT5b suppresses proliferation, induces G1 cell cycle arrest and reduces tumor cell invasion in human glioblastoma multiforme cells. Cancer Lett 273: 164-171, 2009.

10. Lee TK, Man K, Poon RT, et al: Signal transducers and activators of transcription $5 \mathrm{~b}$ activation enhances hepatocellular carcinoma aggressiveness through induction of epithelial-mesenchymal transition. Cancer Res 66: 9948-9956, 2006.

11. Sultan AS, Brim H and Sherif ZA: Co-overexpression of Janus kinase 2 and signal transducer and activator of transcription 5 a promotes differentiation of mammary cancer cells through reversal of epithelial-mesenchymal transition. Cancer Sci 99: 272-279, 2008

12. Darnell JE Jr, Kerr IM and Stark GR: Jak-STAT pathways and transcriptional activation in response to IFNs and other extracellular signaling proteins. Science 264: 1415-1421, 1994.

13. Bromberg J and Darnell JE Jr: The role of STATs in transcriptional control and their impact on cellular function. Oncogene 19: 2468-2473, 2000.

14. Bowman T, Garcia R, Turkson J and Jove R: STATs in oncogenesis. Oncogene 19: 2474-2488, 2000.

15. Gu L, Vogiatzi P, Puhr M, et al: Stat5 promotes metastatic behavior of human prostate cancer cells in vitro and in vivo. Endocr Relat Cancer 17: 481-493, 2010.

16. Tan SH and Nevalainen MT: Signal transducer and activator of transcription 5A/B in prostate and breast cancers. Endocr Relat Cancer 15: 367-390, 2008.
17. Mao Y, Li Z, Lou C and Zhang Y: Expression of phosphorylated Stat5 predicts expression of cyclin D1 and correlates with poor prognosis of colonic adenocarcinoma. Int J Colorectal Dis 26 29-35, 2011

18. Hosui A, Kimura A, Yamaji D, et al: Loss of STAT5 causes liver fibrosis and cancer development through increased TGF- $\{$ beta and STAT3 activation. J Exp Med 206: 819-831, 2009.

19. Peck AR, Witkiewicz AK, Liu C, et al: Loss of nuclear localized and tyrosine phosphorylated Stat5 in breast cancer predicts poor clinical outcome and increased risk of antiestrogen therapy failure. J Clin Oncol 29: 2448-2458, 2011.

20. Bernaciak TM, Zareno J, Parsons JT, et al: A novel role for signal transducer and activator of transcription 5b (STAT5b) in beta1integrin-mediated human breast cancer cell migration. Breast Cancer Res 11: R52, 2009.

21. Tang JZ, Zuo ZH, Kong XJ, et al: Signal transducer and activator of transcription (STAT)-5A and STAT5B differentially regulate human mammary carcinoma cell behavior. Endocrinology 151: 43-55, 2010.

22. Dagvadorj A, Kirken RA, Leiby B, et al: Transcription factor signal transducer and activator of transcription 5 promotes growth of human prostate cancer cells in vivo. Clin Cancer Res 14: $1317-1324,2008$

23. Xiong H, Su WY, Liang QC, et al: Inhibition of STAT5 induces G1 cell cycle arrest and reduces tumor cell invasion in human colorectal cancer cells. Lab Invest 89: 717-725, 2009.

24. Du W, Wang YC, Hong J, et al: STAT5 isoforms regulate colorectal cancer cell apoptosis via reduction of mitochondrial membrane potential and generation of reactive oxygen species. J Cell Physiol: Aug 8, 2011 (Epub ahead of print). doi: 10.1002/ jcp. 22977.

25. Warsch W, Kollmann K, Eckelhart E, et al: High STAT5 levels mediate imatinib resistance and indicate disease progression in chronic myeloid leukemia. Blood 117: 3409-3420, 2011

26. Yamada O, Ozaki K, Furukawa T, et al: Activation of STAT5 confers imatinib resistance on leukemic cells through the transcription of TERT and MDR1. Cell Signal 23: 1119-1127, 2011.

27. Koppikar P, Lui VW, Man D, et al: Constitutive activation of signal transducer and activator of transcription 5 contributes to tumor growth, epithelial-mesenchymal transition, and resistance to epidermal growth factor receptor targeting. Clin Cancer Res 14: 7682-7690, 2008 .

28. Thomas C, Zoubeidi A, Kuruma H, et al: Transcription factor Stat5 knockdown enhances androgen receptor degradation and delays castration-resistant prostate cancer progression in vivo. Mol Cancer Ther 10: 347-359, 2011.

29. Johnstone RW, Ruefli AA and Lowe SW: Apoptosis: a link between cancer genetics and chemotherapy. Cell 108: 153-164, 2002.

30. Reed JC: Dysregulation of apoptosis in cancer. J Clin Oncol 17: 2941-2953, 1999.

31. Ghobrial IM, Witzig TE and Adjei AA: Targeting apoptosis pathways in cancer therapy. CA Cancer J Clin 55: 178-194, 2005. 